\title{
The impact of cardiovascular risk factors on cognition in Hispanics and non-Hispanic whites
}

\author{
Ariana Stickel, ${ }^{1}$ Andrew McKinnon, ${ }^{2}$ John Ruiz, ${ }^{1}$ Matthew D. Grilli, ${ }^{1}$ Lee Ryan, ${ }^{1}$ \\ and Alzheimer's Disease Neuroimaging Initiative ${ }^{3}$ \\ ${ }^{1}$ Department of Psychology, University of Arizona, Tucson, Arizona 85721, USA; ${ }^{2}$ Brain and Mind Centre, University of Sydney, \\ Camperdown, Sydney 2050, Australia
}

\begin{abstract}
Among non-Hispanic whites, cardiovascular risk factors are associated with increased mortality and poorer cognition. Prevalence of cardiovascular risk factors among aging Hispanics is also high and Hispanics generally have poorer access to healthcare, yet they tend to have advantageous cardiovascular disease rates and outcomes and live longer than nonHispanic whites, an epidemiological phenomenon commonly referred to as the Hispanic or Latino health paradox. Although robust data support these ethnic benefits on physical health and mortality, it is unknown if it extends to include cognition resilience advantages in older adulthood. The present study compared relationships between cardiovascular risk and cognition (executive functions and episodic memory) in late middle age and older Hispanics $(n=87)$ and nonHispanic whites $(n=81)$. Participants were selected from the National Alzheimer's Coordinating Center and Alzheimer's Disease Neuroimaging Initiative databases. Hispanics and non-Hispanic white groups were matched on age (50-94 yr, mean age $=72$ yr), education, gender, cognitive status (i.e., cognitively healthy versus mildly cognitively impaired), and apolipoprotein E4 status. History of hypertension and higher body mass index were both associated with poorer executive functions among Hispanics but not non-Hispanic whites. Our findings suggest greater vulnerability to impairments in executive functions among Hispanics with hypertension and obesity, contrary to the notion of a Hispanic health paradox for cognitive aging.
\end{abstract}

The population over the age of $65 \mathrm{yr}$ in the United States continues to grow and become increasingly diverse. By 2030, adults ages 65 and older will outnumber individuals under the age of 18 for the first time in the country's history (United States Census Bureau 2018). Among older adults, Hispanics are one of the fastest growing groups in the United States (Colby and Ortman 2015). Compared to non-Hispanic whites, Hispanics generally experience higher rates of poverty, less education, and less access to medical care (Gallo et al. 2009a) though there is wide variability between Hispanic subgroups (Fenelon et al. 2017). Despite these socioeconomic barriers, numerous studies have demonstrated that Hispanics have lower mortality rates than non-Hispanic whites, a pattern of resilience known as the Hispanic mortality paradox (Abraído-Lanza et al. 1999). The Hispanic mortality paradox is robust, applying to multiple diseases affecting lifespan including end-stage renal disease, cardiovascular disease, and some forms of cancer (for reviews, see Ruiz et al. 2013, 2016). Importantly, a recent meta-analysis of longitudinal studies estimated that averaged across multiple disease domains, Hispanics have a $17.5 \%$ lower mortality rate compared to non-Hispanic groups (Ruiz et al. 2013). This effect is particularly consistent in studies of cardiovascular disease. For example, the majority of studies find that Hispanics live longer than non-Hispanic whites with similar car-

${ }^{3}$ Data used in preparation of this article were obtained from the Alzheimer's Disease Neuroimaging Initiative (ADNI) database (adni. loni.usc.edu). As such, the investigators within the ADNI contributed to the design and implementation of ADNI and/or provided data but did not participate in analysis or writing of this report. A complete listing of ADNI investigators can be found at http://adni.loni.usc .edu/wp-content/uploads/how_to_apply/ADNI_Acknowledgement List.pdf

Corresponding author: ryant@email.arizona.edu

Article is online at http://www.learnmem.org/cgi/doi/10.1101/lm.048470. 118. Freely available online through the Learning \& Memory Open Access option. diovascular diseases including coronary heart disease (for review, see Medina-Inojosa et al. 2014). Further, Hispanics have lower rates of heart disease and strokes than non-Hispanic whites (Ruiz et al. 2016), suggesting protection from some physical health diseases (a Hispanic health paradox) in addition to decreased mortality (the Hispanic mortality paradox).

In the present study, we consider whether the Hispanic health paradox-resilience to the physical effects of cardiovascular risk and disease-may also apply to age-related cognitive decline. We focus on two risk factors-hypertension and increased body fat-that are highly prevalent among both Hispanics and non-Hispanic whites, in order to determine whether they may differentially affect cognitive functioning in these two groups. Hypertension has a $65 \%$ prevalence in adults age $65 \mathrm{yr}$ and older (Go et al. 2013), and obesity, which is commonly defined as body mass index (BMI) greater than or equal to 30 is present in $35 \%$ of the population age $60 \mathrm{yr}$ and older (Ogden et al. 2013). In general, Hispanics tend to have $2 \%-5 \%$ lower prevalence of hypertension across the lifespan compared to non-Hispanic whites (Sorlie et al. 2014; Balfour et al. 2016) but higher rates of obesity, with rates at $37 \%$ and $43 \%$ for Hispanic males and females, respectively (Daviglus et al. 2012). Even among cognitively normal older adults, the presence of cardiovascular risk factors (e.g., hypertension and obesity) is associated with poorer cognitive performance (Raz et al. 2003; Gunstad et al. 2007; Crichton et al. 2014; Bangen et al. 2015; Yeung and Loken Thornton 2017) and increased risk of Alzheimer's and vascular dementia (Meng et al. 2014; for reviews, see Tolppanen et al. 2012; Pedditizi et al. 2016). Although fewer studies have been carried out among Hispanic cohorts, the results suggest that the presence of

(C) 2019 Stickel et al. This article, published in Learning \& Memory, is available under a Creative Commons License (Attribution-NonCommercial 4.0 International), as described at http://creativecommons.org/licenses/by-nc/4.0/. 
cardiovascular risk factors has similar negative effects on cognition among older Hispanics, including increasing risk of dementia (Haan et al. 2003; but see Rodríguez-Saldaña et al. 2002).

Individuals with hypertension (chronic high blood pressure) have poorer processing speed, executive functions, and episodic memory compared to normotensive individuals (for review, see van den Berg et al. 2009). Hypertension most consistently affects episodic memory (van den Berg et al. 2009) and is associated with greater declines in episodic memory functioning over a 20-yr span (Szoeke et al. 2016). Within another cognitive domain, Raz et al. (2003) found that the number of years of hypertension predicted greater numbers of perseverative errors and poorer abstract reasoning on tests of executive function. Perseveration is indicative of poor flexibility and difficulty shifting attention between different elements within a task (Fisk and Sharp 2004).

Among Hispanics, history of hypertension has not been well studied as a predictor of age-related cognitive functioning compared to measurements of blood pressure. For example, higher systolic blood pressure and diastolic blood pressure have been linked to poorer performance on a verbal memory task, but only systolic blood pressure was linked to decreases in psychomotor speed in a large sample of over 9000 individuals (Tarraf et al. 2017). In a longitudinal study of Hispanics, blood pressure did not predict baseline memory performance or change in memory over an average 2.6-yr period, although baseline blood pressure predicted declines on a measure of global cognitive functioning over the same time period (Yaffe et al. 2007). In one study of hypertension, Hispanics with poorly controlled hypertension performed similarly to those with well-controlled hypertension on a verbal memory task but performed worse on a processing speed task (Lamar et al. 2017).

Another common cardiovascular risk factor, obesity, has also been shown to negatively impact attention and memory functioning (Cournot et al. 2006). When present during midlife, obesity is associated with cognitive impairment in later life (for review, see Pedditizi et al. 2016). Increased body fat is most consistently associated with poorer executive functioning (Gunstad et al. 2007; Li et al. 2008; Walther et al. 2010; for review, see Smith et al. 2011). One longitudinal study found that higher BMI at baseline, rather than increases in BMI over a 5-yr period, predicted declines in processing speed and word list learning (Cournot et al. 2006). Conversely, substantially reducing body fat through bariatric surgery resulted in improvements in attention, memory, and executive functions up to $3 \mathrm{yr}$ after surgery (Gunstad et al. 2011; Alosco et al. 2014), suggesting increased body fat and obesity are modifiable risk factors for cognitive impairment.

Although increased body fat and obesity are established predictors of cognitive impairment among non-Hispanic whites, few studies have directly investigated the impact of these factors on cognition among Hispanics. This is particularly striking given the increased prevalence of obesity among this population (Daviglus et al. 2012). In studies of Hispanics with metabolic syndrome, obesity tends to fail to predict cognitive functions when taking other factors into account, including age, gender, education, Hispanic/ Latino subgroup, and depressive symptoms (González et al. 2018). However, in a predominantly Mexican American sample, higher waist-to-hip ratio, a measure of central body fat, was associated with lower hippocampal volumes and greater white matter hyperintensities even when controlling for other cardiovascular factors including cholesterol level, blood pressure, BMI, and glucose and insulin levels (Jagust et al. 2005). Further, these same brain measures predicted the degree of cognitive impairment (cognitively normal versus mild cognitive impairment; Wu et al. 2002).

Taken together, hypertension and increased blood pressure appear to impact cognition negatively among both Hispanics and non-Hispanic whites, although studies differ as to the cogni- tive domain that is affected. It remains unclear as to the impact of obesity and increased body fat among Hispanics, given the relative paucity of studies. Further, few studies have considered whether these cardiovascular risk factors impact cognition differentially among Hispanics and non-Hispanic whites. Comparisons across existing studies described above are exceedingly difficult because cohorts often differ in terms of demographics or educational attainment, as well as other variables that may affect age-related cognitive functioning. To provide evidence relevant to these issues, we investigated the link between cardiovascular health and cognition in the same cohorts of older Hispanic and non-Hispanic whites.

Specifically, the present study compared the impact of hypertension (self-reported history of hypertension) and increased body fat (measured by BMI) on cognitive functioning in late middle age and older Hispanics versus non-Hispanic whites, ages 50-94 yr. Hispanics $(n=87)$ and non-Hispanic whites $(n=81)$ were selected from the National Alzheimer's Coordinating Center (NACC) and Alzheimer's Disease Neuroimaging Initiative (ADNI) databases. Importantly, these groups were carefully matched on age, education, gender, cognitive status (i.e., cognitively healthy versus mild cognitive impairment), and apolipoprotein E4 status. The present study included measures of executive functions (Trail Making Test and Digit Span Backward) and episodic memory (verbal memory delayed recall) as cognitive outcomes. Given the differential rates of hypertension (lower rates) and obesity (higher rates) among Hispanics compared to non-Hispanic whites, we tested these two cardiovascular risk predictors separately. We predicted that history of hypertension and higher BMI would be associated with poorer memory and executive functions in both ethnic groups when controlling for age and education. Additionally, we predicted that Hispanic ethnicity may be protective of cognition, such that the impact of cardiovascular risk factors on cognition would be stronger among non-Hispanic whites, suggesting greater vulnerability, than among Hispanics.

\section{Results}

Hispanics and non-Hispanic white groups were matched on age, education, systolic blood pressure, and diastolic blood pressure $(t s \leq 1.65$, n.s.) as well as gender, cognitive status (i.e., cognitively healthy versus mild cognitive impairment), apolipoprotein $\mathrm{E} 4$ (APOE E4) status (a genetic risk factor for Alzheimer's disease; Farrer et al. 1997), and history of hypertension $\left(\chi^{2} s \leq 1.44\right.$, n.s.). Hispanics had marginally higher BMI than non-Hispanic whites $\left(t_{(164)}=-1.85, P=0.07\right)$. Additionally, among the NACC participants, ethnic groups were matched on antihypertensive medication and lipid-lowering medication treatments $\left(\chi^{2} s \leq 1.53\right.$, n.s.), but Hispanics were marginally more likely to be taking medication for diabetes than non-Hispanic whites $\left(\chi^{2}=3.45, P=0.06\right)$. Unfortunately, there was no or incomplete data on medication use to treat the above cardiovascular risk factors for the majority of ADNI participants. Therefore, we only tested medication differences among NACC participants. See Table 1 for demographics. Given that different verbal memory tests were administered to NACC and ADNI participants (the Wechsler Memory Scale-III or -R Logical Memory subtest and the Rey Auditory Verbal Learning Test, respectively), memory test scores were standardized (transformed to $Z$-scores) separately using the mean and standard deviation of the corresponding NACC sample or the corresponding ADNI sample to create a single delayed verbal memory score across all participants. Other outcomes of interest were Trail Making Test (Trail B raw score divided by Trail A raw score, Trails B/A; Arbuthnott and Frank 2000) and Digit Span Backward raw score. Not all participants completed each cognitive task, so degrees of freedom vary by task (see Materials and Methods for details). 
Table 1. Participant demographics for Hispanics compared to non-Hispanic whites. APOE E4 represents the percentage of E4 carriers (E4 heterozygotes and homozygotes) in each group

\begin{tabular}{|c|c|c|}
\hline & \multicolumn{2}{|c|}{ Ethnicity } \\
\hline & $\begin{array}{l}\text { Hispanics } \\
(n=87)\end{array}$ & $\begin{array}{l}\text { Non-Hispanic whites } \\
\qquad(n=81)\end{array}$ \\
\hline Age (years), $M( \pm$ SEM $)$ & $72.64(0.88)$ & $72.84(1.12)$ \\
\hline Education (years), $M( \pm$ SEM) & $12.59(0.48)$ & $13.56(0.34)$ \\
\hline Body mass index, $M( \pm$ SEM $)$ & $28.18(0.59)$ & $26.69(0.55)$ \\
\hline Systolic blood pressure, $M( \pm$ SEM) & $164.89(16.63)$ & $157.15(19.04)$ \\
\hline Diastolic blood pressure, $M( \pm$ SEM $)$ & $92.24(18.15)$ & $95.60(20.41)$ \\
\hline Gender (\% male) & 29.9 & 38.3 \\
\hline Mild cognitive impairment (\%) & 41.4 & 50.6 \\
\hline$A P O E \mathrm{E} 4(\%)$ & 25.6 & 30.9 \\
\hline History of hypertension (\%) & 60.9 & 59.3 \\
\hline Antihypertensive medication (\%) ${ }^{\mathrm{a}}$ & 58.5 & 68.1 \\
\hline Diabetes medication (\%) ${ }^{\mathrm{a}}$ & 35.8 & 19.1 \\
\hline Lipid lowering medication (\%) & 47.2 & 59.6 \\
\hline
\end{tabular}

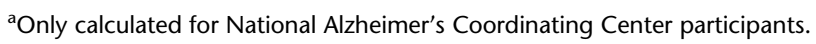

Table 2 lists the number of participants, means, and standard error of the means per ethnic group for each cognitive outcome variable.

Six generalized linear models (GLMs) were performed to separately test the main effects of cardiovascular risk factors (hypertension, BMI) and ethnicity as well as the interaction between cardiovascular risk factor and ethnicity, predicting the three cognitive outcome measures. Three GLMs were conducted for hypertensive status (categorical yes/no variable) and three were conducted for BMI (linear variable). All GLMs included age and education as linear covariates. Table 3 lists the overall $F$-statistics for the six models. Predictors and covariates are listed for those models that met significance or were marginally significant. Results shown are $F$-statistics from GLMs with the exception of $t$-statistics from parameter estimates with robust standard errors in which violations of homoskedasticity occurred.

\section{Hypertension}

Of the three GLMs that included hypertension status as a predictor, the overall models for delayed verbal memory and backward digit span were significant, while the overall model for Trails B/ A was marginally significant. However, specific predictors for each cognitive test varied. For delayed verbal memory, increasing age dominated as the major predictor of poorer performance $\left(F_{(1,157)}=15.92, P<0.001\right)$, while higher education was marginally associated with better memory $\left(F_{(1,157)}=3.75, P=0.06\right)$. Delayed verbal memory was not predicted by hypertensive status, ethnicity, nor their interaction ( $F \mathrm{~s} \leq 1.08$, n.s.). For Trails B/A, somewhat surprisingly, no main effects of predictors nor covariates successfully predicted performance $(F s \leq 2.13$, n.s.). However, we observed a trend for the interaction between ethnicity and hypertension $\left(F_{(1,154)}=3.55, P=0.06\right)$, such that Hispanics with hypertension had higher Trails $\mathrm{B} / \mathrm{A}$ scores (a ratio of time to complete Trails $B$ relative to Trails A, indicating poorer performance), compared to normotensive Hispanics $\left(t_{(78)}=-2.24, P<0.05\right)$ as well as compared to non-Hispanic whites with hypertension $\left(t_{(93)}=-1.99, P<0.05\right.$; see Fig. 1$)$. No other group differences were detected ( $t \leq \leq 1.58$, n.s.). Finally, with regard to Digit Span Backward, Hispanics showed overall poorer performance than non-Hispanic whites, indicated by a main effect of ethnicity ( $t=$ 4.53, $P<0.001)$. Additionally, higher education was associated with better performance on the task $(t=3.13, P<0.01)$. No other predictors (hypertension, the interaction between hypertension and ethnicity) nor the age covariate predicted Digit Span Backward performance ( $t \mathrm{~s} \leq 1.44$, n.s.).

\section{Body mass index}

The pattern of results for BMI were generally similar to the hypertension models. For GLMs including BMI as a predictor, only the models for delayed verbal memory and Digit Span Backward were significant. The overall model for Trails B/A was not significant $\left(F_{(5,152)}=1.48\right.$, n.s. $)$. Therefore, we did not interpret its predictors. As with the above GLMs, delayed verbal memory was predicted by age and education. Specifically, younger age $\left(F_{(1,155)}=15.65, P\right.$ $<0.001)$ and higher years of education $\left(F_{(1,155)}=4.15, P<0.05\right)$ were linked to better-delayed verbal memory scores. The main effects of BMI, ethnicity, and the interaction between BMI and ethnicity did not approach significance ( $F s \leq 1.90$, n.s.). Consistent with the earlier analysis, Hispanics had significantly poorer Digit Span Backward scores than non-Hispanic whites $(t=-2.19, P<$ 0.05). Further, the interaction between ethnicity and BMI significantly predicted Digit Span Backward $(t=3.25, \quad P=0.001)$. Specifically, higher BMI was significantly associated with poorer performance on Digit Span Backward among Hispanics (Pearson's $r(60)=-0.34, P<0.01$ ) but was not significantly related to performance among non-Hispanic whites (Pearson's $r(62)=$ 0.17 , n.s.). Figure 2 shows both effects identified in this GLM -

Table 2. Mean (standard error of the mean) and numbers of participants $(N)$ for each cognitive task per ethnicity group

\begin{tabular}{|c|c|c|c|c|}
\hline & \multicolumn{4}{|c|}{ Ethnicity } \\
\hline & \multicolumn{2}{|c|}{ Hispanics } & \multicolumn{2}{|c|}{ Non-Hispanic whites } \\
\hline & $N$ & Mean & $N$ & Mean \\
\hline Delayed verbal memory $z$-score, $M( \pm$ SEM) & 82 & $0.06(0.10)$ & 81 & $-0.06(0.12)$ \\
\hline Trails $\mathrm{B} / \mathrm{A}$ raw, $M( \pm \mathrm{SEM})$ & 79 & $3.23(0.15)$ & 81 & $3.00(0.13)$ \\
\hline Digit span backward raw, $M( \pm S E M)$ & 61 & $4.42(0.23)$ & 67 & $6.23(0.29)$ \\
\hline
\end{tabular}


Table 3. F-statistics for each of six models (in bold) and predictor/covariate factors for significant or marginally significant models

\begin{tabular}{|c|c|c|c|}
\hline & Delayed memory & Trails B/A & Digit span backward \\
\hline \multicolumn{4}{|l|}{ Hypertension } \\
\hline $\begin{array}{l}\text { Overall model } \\
\text { Hypertension }\end{array}$ & $\begin{array}{l}\boldsymbol{F}_{(\mathbf{5}, \mathbf{1 5 7})}=\mathbf{5 . 0 3}^{* * *} \\
F_{(1,15)}=0.42\end{array}$ & $\begin{array}{l}\boldsymbol{F}_{(\mathbf{5}, \mathbf{1 5 4})}=\mathbf{1 . 9 0 ^ { \dagger }} \\
F_{(1,154)}=2.13\end{array}$ & $\begin{array}{l}\boldsymbol{F}_{(\mathbf{5}, \mathbf{1 2 2})}=\mathbf{8 . 2 3}^{* * *} \\
t=0.73\end{array}$ \\
\hline Ethnicity & $F_{(1,157)}=1.08$ & $F_{(1,154)}=0.68$ & $t=4.53^{* \star *}$ \\
\hline Hypertension $\times$ ethnicity & $F_{(1,157)}=0.18$ & $F_{(1,154)}=3.55^{\dagger}$ & $t=-1.44$ \\
\hline Age & $F_{(1,157)}=15.92^{* * *}$ & $F_{(1,154)}=1.33$ & $t=-0.79$ \\
\hline Education & $F_{(1,157)}=3.75^{\dagger}$ & $F_{(1,154)}=1.19$ & $t=3.13^{* *}$ \\
\hline \multicolumn{4}{|l|}{ Body mass index } \\
\hline Overall model & $F_{(5,155)}=5.61^{* * *}$ & $F_{(5,152)}=1.48$ & $F_{(5,120)}=9.18^{\star \star *}$ \\
\hline Body mass index & $F_{(1,155)}=0.16$ & $(0,102)$ & $t=-3.19^{\star *}$ \\
\hline Ethnicity & $F_{(1,155)}=1.37$ & - & $t=-2.19^{\star}$ \\
\hline Body mass index $x$ ethnicity & $F_{(1,155)}=1.90$ & - & $t=3.25^{\star \star}$ \\
\hline Age & $F_{(1,155)}=15.65^{\star \star *}$ & - & $t=-0.80$ \\
\hline Education & $F_{(1,155)}=4.15^{*}$ & - & $t=2.91^{\star \star}$ \\
\hline
\end{tabular}

$t$-Statistics from robust standard errors are included in place of $F$-statistics for both models predicting Digit Span Backward scores, in which there was a violation of homoskedasticity. $\left(^{\dagger}\right) P<0.10,\left(^{*}\right) P<0.05,\left({ }^{* *}\right) P<0.01,\left({ }^{* *}\right) P<0.001$.

overall, scores for non-Hispanic whites are higher than those for Hispanics, and scores among Hispanics decrease as BMI increases.

\section{Discussion}

The present study investigated the role of cardiovascular risk factors (i.e., hypertension and obesity) on cognition among late middle-aged and older Hispanics compared to a group of carefully matched non-Hispanic whites. To summarize the results, only age and education predicted scores on a delayed memory test-hypertension and BMI did not impact memory scores for either ethnic group. In contrast, both hypertension and higher BMI were associated with poor executive functions. However, contrary to our hypothesis, these cardiovascular risk factors were detrimental among Hispanics but not non-Hispanic whites. Our findings suggest that Hispanics may be more vulnerable to cognitive decline in the presence of cardiovascular risk factors rather than being protected against cognitive decline, as may be expected by the Hispanic health paradox.

Unlike previous studies of predominantly non-Hispanic white samples (Raz et al. 2003; Szoeke et al. 2016; for review, see van den Berg et al. 2009), hypertension did not impact cognition among the non-Hispanic white group in this study, nor across the two ethnic groups combined. Rather, we observed a marginal interaction with poorer performance on a task switching test, Trails B/A, among hypertensive Hispanics compared to normotensive Hispanics and non-Hispanic whites. Given that this interaction was trending and that with more power non-Hispanic whites might also show a negative impact of hypertension on task switching, further research is warranted. Considering that our simple effects tests detected differences between normotensive and hypertensive Hispanics but not between respective non-Hispanic whites, with more power we might expect a main effect of hypertension in addition to interaction with Hispanics being more negatively impacted than non-Hispanic whites. The notion of greater susceptibility among Hispanics is consistent with the significant interaction observed on working memory.

On average, Hispanics showed poorer performance on a working memory test (the Digit Span Backward) compared to non-Hispanic whites, even when age and education were included as covariates. This overall difference in performance could be attributed to cultural biases in digit span tests due to differences in linguistic chunking strategies among Hispanics which is independent of English or Spanish test administration (LaRue et al. 1999). However, more critical to our study was the finding that higher BMI differentially predicted poorer performance on a working memory test among Hispanic individuals, but not non-Hispanic whites. Again, this suggests that a modifiable risk factor may

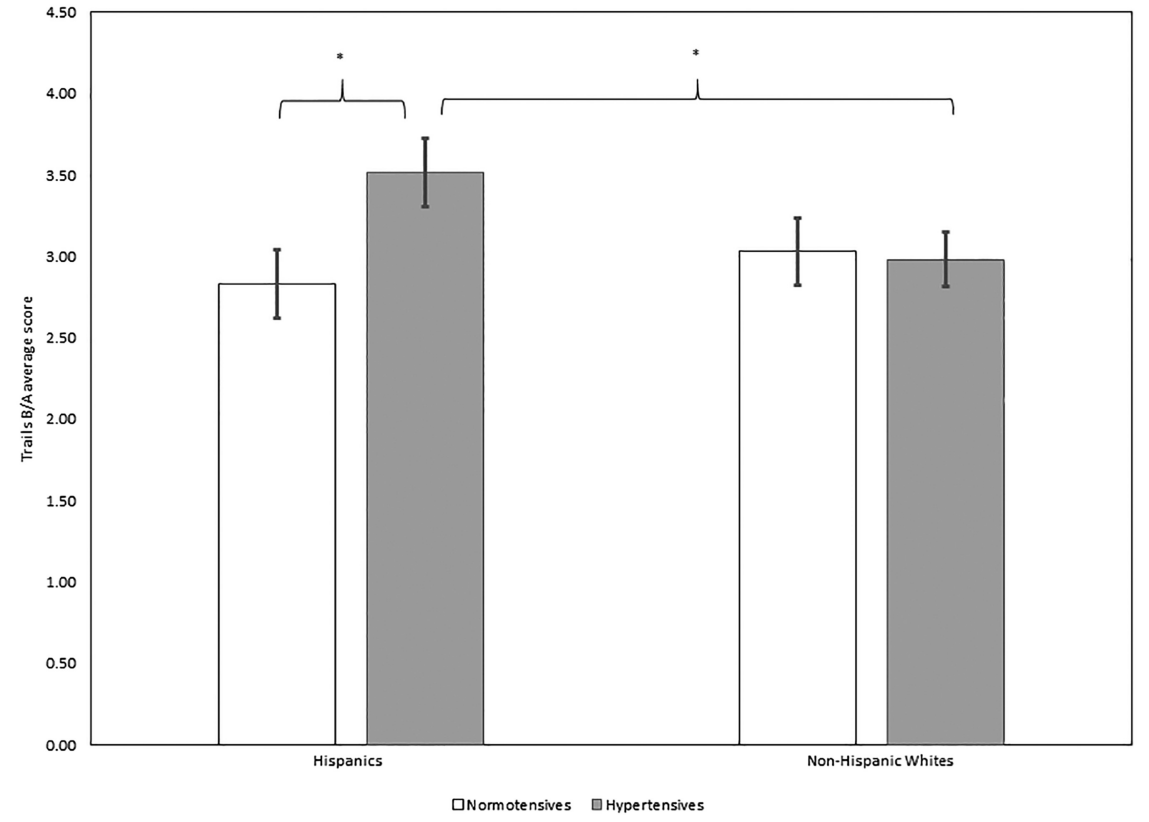

Figure 1. Average Trails B/A scores for normotensive and hypertensive Hispanics (left) and normotensive and hypertensive non-Hispanic whites (right). Hypertensive Hispanics had higher average Trails B/A scores compared to normotensive Hispanics and hypertensive non-Hispanic whites. Note, the overall interaction was marginal $\left(F_{(1,154)}=3.55, P=0.06\right)$. Higher Trails $\mathrm{B} / \mathrm{A}$ scores reflect poorer task switching abilities. Normotensives are represented in white. Hypertensives are represented in gray. $\left(^{*}\right) P<0.05$. 


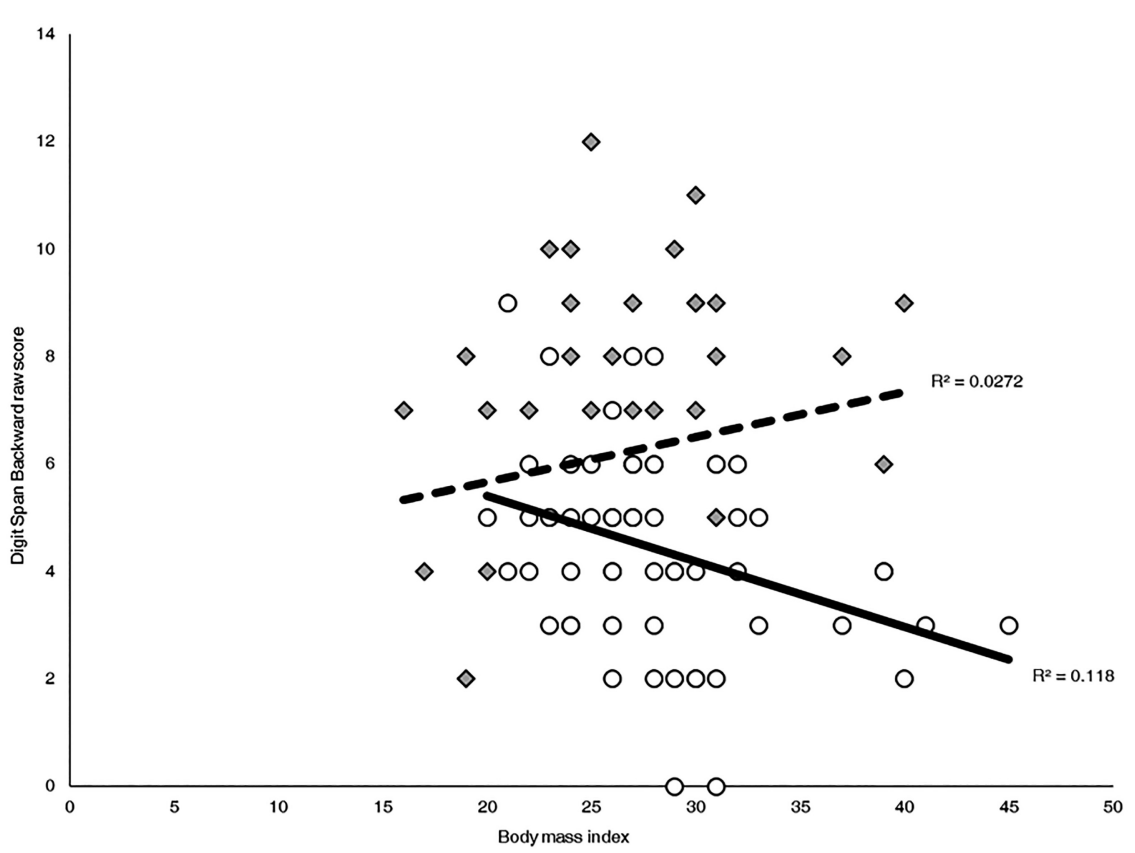

Figure 2. Pearson correlations and percent variance explained $\left(R^{2}\right)$ between Digit Span Backward raw scores and BMI per ethnic group. Among Hispanics, higher BMI was associated with lower Digit Span Backward scores, $(r(60)=-0.34, P<0.01)$. BMI was not associated with scores among non-Hispanic whites $(r(62)=.17, \mathrm{n} . \mathrm{s}$.$) . Hispanics are represented by open dots and a solid line. Non-Hispanic$ whites are represented by shaded diamonds and a dashed line.

negatively impact cognition to a greater extent among Hispanics than non-Hispanic whites.

Haan et al. (2003) suggested that cardiovascular-related health factors (e.g., diabetes and stroke) rather than the apolipoprotein E4 status increase prevalence of dementia to a greater extent among Mexican Americans than non-Hispanic whites. Our findings provide some support for the notion that cardiovascular risk factors may be more detrimental to Hispanics than non-Hispanic whites. It is important to note that Hispanics are less likely to be treated for hypertension and are less likely to achieve well-managed hypertension as compared to non-Hispanic whites (for review, see Balfour et al. 2016), and this may contribute to greater susceptibility to cognitive impairment among Hispanics with hypertension. Rates of Alzheimer's disease among Hispanics are similar to (Haan et al. 2003) or greater than rates among non-Hispanic whites (Gurland et al. 1999), and differential impact of cardiovascular risk factors on cognition may be contributing to the latter. Furthermore, cardiovascular risk factors are associated with in- creased risk of vascular dementia which may also affect higher proportions of Hispanics than non-Hispanic whites (Fitten et al. 2001). Both vascular dementia and Alzheimer's disease can include impairments in episodic memory and executive functioning. However, impairments in episodic memory are a critical diagnostic feature of typically progressing Alzheimer's disease but not necessarily vascular dementia whereas impairments across several subdomains of executive functioning are more common in early stages of vascular dementia than in Alzheimer's disease (Graham et al. 2004). In the present study, episodic memory was not differentially impacted by cardiovascular risk factors, regardless of ethnicity. As noted earlier, hypertension has been consistently associated with poorer episodic memory (Szoeke et al. 2016; for review, see van den Berg et al. 2009) and somewhat less consistently with poorer executive functions (Raz et al. 2003). However, in the present study, hypertension did not have a direct effect on either cognitive domain. Hypertension has been suggested to influence cognition through several mechanisms, including reduced cerebral blood flow and loss of blood vessel elasticity which increases risk of stroke (Kannel et al. 1981) and leads to small vessel damage which, in turn, is associated with increases in white matter hyperintensities on magnetic resonance imaging (MRI; Carmelli et al. 1999; Raz et al. 2003; Salat 2014). Obesity, on the other hand, is most consistently associated with executive functions (Gunstad et al. 2007; Li et al. 2008; Walther et al. 2010; for review, see Smith et al. 2011) and to a lesser extent episodic memory. Obesity is thought to impact cognition by increasing the production of proinflammatory markers (e.g., interleukin-6, TNF- $\alpha$ ), many of which cross the bloodbrain barrier and can lead to brain deterioration if sustained (Fuentes et al. 2013; Verstynen et al. 2013; Kiliaan et al. 2014). Additionally, obesity reduces blood flow to the brain (Birdsill et al. 2013; Bagi et al. 2014).

Within the domain of executive functioning, it is important to note that hypertension predicted task switching while increased BMI predicted working memory. Hypertension has been shown to impact specific aspects of executive functions in other studies as well. For example, Raz et al. (2003) found that hypertension was

Table 4. Numbers of participants (divided by ethnicity) with cognitive data broken down by task

\begin{tabular}{|c|c|c|c|c|c|c|}
\hline & \multicolumn{2}{|c|}{ NACC } & \multicolumn{2}{|c|}{ ADNI } & \multicolumn{2}{|c|}{ Total } \\
\hline & Hispanic & NHW & Hispanic & NHW & Hispanic & NHW \\
\hline \multicolumn{7}{|l|}{ Episodic memory } \\
\hline RAVLT & - & - & 34 & 34 & 34 & 34 \\
\hline Logical memory & 48 & 47 & - & - & 48 & 47 \\
\hline \multicolumn{7}{|l|}{ Executive functions } \\
\hline Trail B & 45 & 47 & 34 & 34 & 79 & 81 \\
\hline Digit Span Backward & 48 & 47 & 13 & 20 & 61 & 67 \\
\hline \multicolumn{7}{|l|}{ Processing speed } \\
\hline Trail A & 52 & 47 & 34 & 34 & 86 & 81 \\
\hline
\end{tabular}

NACC, National Alzheimer's Coordinating Center; ADNI, Alzheimer's Disease Neuroimaging Initiative; NHW, non-Hispanic White; RAVLT, Rey Auditory Verbal Learning Test. 
associated with greater numbers of perseverative errors, an indicator of poor cognitive flexibility, but was unrelated to working memory. Our data support the notion of distinct mechanisms by which hypertension versus increased BMI impact cognition. Miyake and Friedman's (2012) and Friedman and Miyake's (2017) models of executive functioning frame task switching and updating working memory as related but distinct subdomains of executive functions which may rely on different neurological pathways. This model has been supported by a recent diffusion MRI study (Bettcher et al. 2016), demonstrating that subdomains of executive functions are associated with distinct patterns of white matter integrity-both task switching and working memory performance were associated with diffusion in the cingulum, but diffusion in the corpus callosum specifically predicted task switching abilities.

Although the present study did not directly compare the impact of hypertension to obesity, we found an interaction between ethnicity and increased BMI on working memory, and a similar pattern between ethnicity and hypertension on task switching. Among samples of predominantly non-Hispanic whites and even predominantly Hispanic samples, hypertension tends to be a more robust predictor of cognition than obesity (Nilsson and Nilsson 2009; Levin et al. 2014; Yesavage et al. 2014). For example, among the ethnically diverse Northern Manhattan Study sample, blood pressure was the most robust cardiovascular predictor of executive functioning, even after controlling for other cardiovascular risk factors, whereas obesity failed to predict cognition when other cardiovascular risk factors were taken into account.

Other factors not accounted for in the present study may have impacted our results. First, similar to previous studies (Zahodne et al. 2015), our sample included participants with and without a diagnosis of mild cognitive impairment (all subtypes), carefully matching ethnic groups on the proportions of cognitively healthy to mildly cognitively impaired. Mild cognitive impairment is characterized in multiple ways, and there is an even greater dearth of information on mild cognitive impairment among Hispanics. There is some evidence to suggest that mild cognitive impairment among Hispanics is distinct from non-Hispanic whites. At a neurological level, Hispanics with mild cognitive impairment have greater hippocampal volumes compared to non-Hispanic whites, yet ethnic groups do not differ in hippocampal volumes among those with a dementia diagnosis (DeCarli et al. 2008). More research is needed to clarify whether Hispanics are better able to maintain hippocampal volumes in the early stages of possible neurodegeneration. In the present study, the cognitive status may have further moderated the impact of cardiovascular risk on executive functions. However, due to our relatively small sample sizes, we did not examine the three-way interaction between cognitive status, ethnicity, and cardiovascular risk factors on cognition.

Second, the age at which cardiovascular risk factors are acquired impacts their effect on cognition. Among non-Hispanic whites, midlife hypertension and midlife obesity are both associated with increased risk of Alzheimer's disease, yet onset of these two cardiovascular risk factors in later life may be protective against cognitive impairment (Smith et al. 2011; Memel et al. 2016; Pedditizi et al. 2016; Corrada et al. 2017). In the present study, the lack of an effect of cardiovascular risk among non-Hispanic whites may reflect variability in onset of risk factors among a wide age range of older adults. It is unclear as to whether or not this pattern is true for Hispanics. Interestingly, in a study of metabolic syndrome, the presence of multiple cardiovascular risk factors among Hispanics was predictive of episodic memory only among late middle age individuals (González et al. 2018). In contrast, executive functions were negatively impacted across all age groups in the presence of metabolic syndrome (González et al. 2018), which is consistent with the present finding of an association with executive functions but not memory.
Third, although the impact of cardiovascular risk factors is likely mediated by changes in brain structure and function, particularly in the context of aging, other factors may be overshadowing and/or moderating the direct impact of single risk factors. For this reason, measures of brain structure may serve as a better predictor of cognition than the risk factors themselves. For example, among both Hispanics and non-Hispanic whites, total brain volumes have been shown to mediate the relationship between age and executive functions (Mungas et al. 2009); that is, age-associated changes in total brain volumes better-predicted changes in executive functions than age alone. Regarding cardiovascular risk factors and the brain, obesity has been associated with smaller hippocampal volumes and greater white matter hyperintensities among Mexican Americans (Jagust et al. 2005), and Hispanics may be more susceptible to white matter hyperintensities in the presence of high blood pressure than non-Hispanic whites (Marcus et al. 2011). Both lower hippocampal volumes and larger white matter hyperintensity volumes have been linked to poorer cognitive functioning among Hispanics (Wu et al. 2002; DeCarli et al. 2008). However, it is unclear if such brain measures predict cognition equally (DeCarli et al. 2008), to a lesser extent (Zahodne et al. 2015), or to a greater extent among Hispanics compared to non-Hispanic whites. Given the findings from the present study suggesting a Hispanic cognitive disadvantage in the presence of cardiovascular risk factors, further investigation into brain structure as a possible mediator of the impact of cardiovascular risk factors on cognition is warranted.

Fourth, differences between NACC and ADNI databases could potentially limit the generalizability of our findings. The ADNI study, in particular, has enriched its sample to include more individuals with mild cognitive impairment. Although mild cognitive impairment diagnostic criteria may slightly differ between databases (see Weintraub et al. 2009 and adni.loni.usc.edu/study-design), we matched and selected approximately equal numbers Hispanic and non-Hispanic whites from NACC as well as from ADNI, in an attempt to minimize confounds of database. As mentioned above, data on other cardiovascular risk factors (e.g., diabetes and hyperlipidemia) were limited. The competing and combined impacts of hypertension, obesity, and other cardiovascular risk factors on cognition among Hispanics compared to non-Hispanics whites remains to be well characterized.

Finally, the country of origin may also impact the outcomes of cognitive studies. Our study included Hispanic individuals from the NACC and ADNI data sets who identified their race as White. Among our NACC participants, Hispanics predominantly identified Mexico as their country of origin. Country of origin/ancestry was unknown for participants from the ADNI sample. Studies with Hispanics from a wide variety of countries of origin have reported differences in susceptibility for cognitive impairment based on country of origin. Specifically, Puerto Ricans and Dominicans seem to be at greater risk of cognitive impairment compared to Hispanics of other descents (González et al. 2015; Marquine et al. 2018). We do not know whether these Hispanic subgroups differ in risk of cognitive impairment due to differences in sensitivity to cardiovascular risk factors. As noted above, our sample was also distinct from other studies in that our Hispanic and non-Hispanic white samples were matched on age and educational status. Among non-Hispanic whites, higher education is protective against cognitive decline with age (Tucker and Stern 2011), and education is likely protective to cognition among Hispanics as well (Levin et al. 2014). The Hispanics in our sample tended to be more highly educated than Hispanics in other samples of aging (e.g., Haan et al. 2016), and this may be reflective of the White racial identification of our group. Of note, even though our Hispanic sample was matched with non-Hispanic whites on years of education, quality of education may differ between ethnic groups as it 
does with other racial groups (Glymour and Manly 2008), and it may have impacted susceptibility to poorer cognitive functioning. Although the present study did not observe a Hispanic health paradox protection for cognition, Hispanics may be protected from the downstream effects of cognitive impairment. For example, Helzner et al. (2008) observed lower mortality rates among Hispanics compared to non-Hispanic whites who developed Alzheimer's disease while enrolled in a longitudinal study. One possible explanation is that cultural factors common to Hispanics (e.g., values for collectivism and family) may be more effective in building resilience in the presence of disease rather than protecting against disease altogether. Such cultural factors have been hypothesized as the mechanisms underlying the Hispanic health and mortality paradoxes (Ruiz et al. 2016, 2018). Consistent with this notion, the strength of the Hispanic health paradox is attenuated by acculturation-higher adherence to American culture is associated with less resilience to disease (Gallo et al. 2009b). With regard to resilience in the context of cognitive aging, poorer cognition may be less detrimental to daily functioning among Hispanics than among non-Hispanic whites. Recent data from the same sample included in the present study suggest that memory and processing speed, but not executive functions, predicted ability to complete instrumental activities of daily living (e.g., paying bills; Stickel et al. in prep.). Specifically, poorer memory predicted poorer activities of daily living functioning equally among Hispanics and non-Hispanic whites, but only non-Hispanic whites showed poorer functioning in the presence of poorer processing speed. The latter finding suggests that even in the context of mildly poor cognitive functioning, everyday functioning is maintained. However, other studies have observed similar links between cognitive functioning and everyday functioning among Hispanics and non-Hispanic whites or even greater disadvantages among Hispanics rather than Hispanic protection (Tomaszewski Farias et al. 2004; Tarraf et al. 2018). Education can have downstream effects on functioning, including among Hispanics (Haan et al. 2016), and differences in education levels may account for the mixed findings. Other studies control for lower education among Hispanics (Tomaszewski Farias et al. 2004; Tarraf et al. 2018) whereas our ethnic groups were matched on this key factor in addition to controlling for it.

In sum, the present study provides a nuanced view of the impact of cardiovascular health on cognition among older Hispanics and non-Hispanic whites. We examined the impact of hypertension and BMI separately on a variety of neuropsychological measures in a well-matched sample of Hispanics and non-Hispanic whites. We found that executive functions were negatively impacted by hypertension and obesity only among Hispanics, and not among non-Hispanic whites. This finding contradicts the notion of a Hispanic health paradox for cognition. Instead, our findings suggest greater vulnerability to cognitive decline in the presence of cardiovascular risks of Hispanics compared to non-Hispanic whites. Importantly, our data are limited to Hispanics who identified their race as White and the majority of whom claimed Mexican ancestry. Understanding whether these patterns apply to other racial groups and nationalities of Hispanics is a necessary next step.

\section{Materials and Methods}

\section{Participants}

A total of 202 participants ages 50-94 yr were selected from the NACC $(n=134 ; \quad$ https://www.alz.washington.edu/ $)$ and the $\mathrm{ADNI}^{4}(n=68)$ databases. Across both databases, participants' base-

${ }^{4}$ Data used in the preparation of this article were obtained from the Alzheimer's Disease Neuroimaging Initiative (ADNI) database (adni.loni.usc.edu). The ADNI line examinations began between 2005 and 2015. The impact of risk factors for cognitive impairment on brain structure and cognition can vary by race (DeCarli et al. 2008; Glymour and Manly 2008; Zahodne et al. 2015, 2017). Therefore, we selected Hispanics who self-identified their race as white $(n=101)$, in order to reduce potential confounds of race. Participants included cognitively healthy individuals and those with a diagnosis of mild cognitive impairment who self-reported their ethnicity as white or non-Hispanic. From these participants, 168 (NACC $^{5}: n=100$; ADNI: $n=68$ ) had cognitive testing data. Of the 168 participants, 36 Hispanics and 41 non-Hispanic whites were diagnosed with mild cognitive impairment. See Table 1 and Results section above for more demographic comparisons between ethnic groups.

\section{Neuropsychological measures}

Both NACC and ADNI primarily administer the Uniform Data Set (Weintraub et al. 2009, 2018; http://www.adni-info.org/Scientists/ CognitiveTesting.html). Tests included in the Uniform Data Set and used in the present study were the Wechsler Memory Scale (WMS)-III or -R Logical Memory Story-A Immediate and Delayed Recall (Wechsler 1987), WMS-R Digit Span Backward, and Trail Making Test (Parts A and B; Reitan 1958). The Uniform Data Set was translated in Spanish in multiple variations to accommodate cultural/national differences between various Hispanic groups (Acevedo et al. 2009). The Rey Auditory Verbal Learning Test (RAVLT) was also collected, specifically for ADNI participants. See Table 4 for breakdown of numbers of Hispanics and nonHispanic whites with cognitive data per task.

\section{Cardiovascular risk measures}

Hypertensive status was determined by self-reported history of hypertension diagnosis and/or indication of hypertension in medical records. Systolic and diastolic blood pressure measurements were collected at baseline for participants. BMI was calculated using the following formula: (weight (lbs) $\times 703$ )/height (inches) ${ }^{2}$. Height and weight measurements were collected at NACC and ADNI study visits.

\section{Statistical analysis}

To test the separate impacts of hypertension and obesity and their interactions with ethnicity on cognition, two series of GLMs were performed in SPSS (Windows, Version 25.0). Each series included three GLMs that predicted Trails B/A, Digit Span Backward, and delayed verbal memory scores. In other words, a total of six GLMs which included the main effects of cardiovascular risk factor and ethnicity, the interaction between cardiovascular risk factor and ethnicity, and controlled for age and education were performed. Results presented above are $F$-statistics from GLMs with the exception of $t$-statistics from parameter estimates with robust standard errors in which there was a violation of homoskedasticity.

\section{Acknowledgments}

The NACC database is funded by National Institute on Aging (NIA)/National Institutes of Health (NIH) Grant U01 AG016976. NACC data are contributed by the NIA-funded ADCs: P30 AG019610 (PI Eric Reiman, MD), P30 AG013846 (PI Neil Kowall, MD), P50 AG008702 (PI Scott Small, MD), P50 AG025688 (PI Allan Levey, MD, PhD), P50 AG047266 (PI Todd Golde, MD, PhD), P30 AG010133 (PI Andrew Saykin, PsyD), P50 AG005146 (PI Marilyn Albert, PhD), P50 AG005134 (PI Bradley Hyman,

was launched in 2003 as a public-private partnership, led by Principal Investigator Michael W. Weiner, MD. The primary goal of ADNI has been to test whether MRI, positron emission tomography, other biological markers, and clinical and neuropsychological assessment can be combined to measure the progression of mild cognitive impairment and early Alzheimer's disease. For up-to-date information, see www.adni-info.org.

${ }^{5} \mathrm{NACC}$ participants selected for this analysis were from $10 \mathrm{ADCs}$. 
MD, PhD), P50 AG016574 (PI Ronald Petersen, MD, PhD), P50 AG005138 (PI Mary Sano, PhD), P30 AG008051 (PI Thomas Wisniewski, MD), P30 AG013854 (PI M. Marsel Mesulam, MD), P30 AG008017 (PI Jeffrey Kaye, MD), P30 AG010161 (PI David Bennett, MD), P50 AG047366 (PI Victor Henderson, MD, MS), P30 AG010129 (PI Charles DeCarli, MD), P50 AG016573 (PI Frank LaFerla, PhD), P50 AG005131 (PI James Brewer, MD, PhD), P50 AG023501 (PI Bruce Miller, MD), P30 AG035982 (PI Russell Swerdlow, MD), P30 AG028383 (PI Linda Van Eldik, PhD), P30 AG053760 (PI Henry Paulson, MD, PhD), P30 AG010124 (PI John Trojanowski, MD, PhD), P50 AG005133 (PI Oscar Lopez, MD), P50 AG005142 (PI Helena Chui, MD), P30 AG012300 (PI Roger Rosenberg, MD), P30 AG049638 (PI Suzanne Craft, PhD), P50 AG005136 (PI Thomas Grabowski, MD), P50 AG033514 (PI Sanjay Asthana, MD, FRCP), P50 AG005681 (PI John Morris, MD), P50 AG047270 (PI Stephen Strittmatter, MD, PhD). Data collection and sharing for this project was funded by the Alzheimer's Disease Neuroimaging Initiative (ADNI) (National Institutes of Health Grant U01 AG024904) and DOD ADNI (Department of Defense award number W81XWH-12-2-0012). ADNI is funded by the National Institute on Aging, the National Institute of Biomedical Imaging and Bioengineering, and through generous contributions from the following: AbbVie, Alzheimer's Association; Alzheimer's Drug Discovery Foundation; Araclon Biotech; BioClinica, Inc.; Biogen; Bristol-Myers Squibb Company; CereSpir, Inc.; Cogstate; Eisai Inc.; Elan Pharmaceuticals, Inc.; Eli Lilly and Company; EuroImmun; F. Hoffmann-La Roche Ltd and its affiliated company Genentech, Inc.; Fujirebio; GE Healthcare; IXICO Ltd.; Janssen Alzheimer Immunotherapy Research and Development, LLC.; Johnson and Johnson Pharmaceutical Research and Development LLC.; Lumosity; Lundbeck; Merck and Co., Inc.; Meso Scale Diagnostics, LLC.; NeuroRx Research; Neurotrack Technologies; Novartis Pharmaceuticals Corporation; Pfizer Inc.; Piramal Imaging; Servier; Takeda Pharmaceutical Company; and Transition Therapeutics. The Canadian Institutes of Health Research is providing funds to support ADNI clinical sites in Canada. Private sector contributions are facilitated by the Foundation for the National Institutes of Health (www.fnih.org). The grantee organization is the Northern California Institute for Research and Education, and the study is coordinated by the Alzheimer's Therapeutic Research Institute at the University of Southern California. ADNI data are disseminated by the Laboratory for Neuro Imaging at the University of Southern California.

\section{References}

Abraído-Lanza AF, Dohrenwend BP, Ng-Mak DS, Turner JB. 1999. The Latino mortality paradox: a test of the "salmon bias" and healthy migrant hypotheses. Am J Public Health 89: 1543-1548. doi:10.2105/AJPH.89.10 .1543

Acevedo A, Krueger KR, Navarro E, Ortiz F, Manly JJ, Padilla-Vélez MM, Weintraub S, López OL, Mungas D. 2009. The Spanish translation and adaptation of the uniform data set of the National Institute on Aging Alzheimer's Disease Centers. Alzheimer Dis Assoc Disord 23: 102-109. doi:10.1097/WAD.0b013e318193e376

Alosco ML, Galioto R, Spitznagel MB, Strain G, Devlin M, Cohen R, Crosby RD, Mitchell JE, Gunstad J. 2014. Cognitive function after bariatric surgery: evidence for improvement 3 years after surgery. Am J Surg 207: 870-876. doi:10.1016/j.amjsurg.2013.05.018

Arbuthnott K, Frank J. 2000. Trail making test, part B as a measure of executive control: validation using a set-switching paradigm. J Clin Exp Neuropsychol 22: 518-528. doi:10.1076/1380-3395(200008)22:4;1-0; FT518

Bagi Z, Broskova Z, Feher A. 2014. Obesity and coronary microvascular disease-implications for adipose tissue-mediated remote inflammatory response. Curr Vasc Pharmacol 12: 453-461. doi:10.2174/ 1570161112666140423221843

Balfour PCJ, Ruiz JM, Talavera GA, Allison MA, Rodriguez CJ. 2016. Cardiovascular Disease in Hispanics/Latinos in the United States. J Lat Psychol 4: 98-113. doi:10.1037/lat0000056

Bangen KJ, Gu Y, Gross AL, Schneider BC, Skinner JC, Benitez A, Sachs BC, Shih R, Sisco S, Schupf N, et al. 2015. Relationship between type 2 diabetes mellitus and cognitive change in a multiethnic elderly cohort. $J$ Am Geriatr Soc 63: 1075-1083. doi:10.1111/jgs.13441
Bettcher BM, Mungas D, Patel N, Elofson J, Dutt S, Wynn M, Watson CL, Stephens M, Walsh CM, Kramer JH. 2016. Neuroanatomical substrates of executive functions: Beyond prefrontal structures. Neuropsychologia 85: 100-109. doi:10.1016/j.neuropsychologia.2016.03.001

Birdsill AC, Carlsson CM, Willette AA, Okonkwo OC, Johnson SC, Xu G, Oh JM, Gallagher CL, Koscik RL, Jonaitis EM, et al. 2013. Low cerebral blood flow is associated with lower memory function in metabolic syndrome. Obesity 21: 1313-1320. doi:10.1002/oby.20170

Carmelli D, Swan GE, Reed T, Wolf PA, Miller BL, DeCarli C. 1999. Midlife cardiovascular risk factors and brain morphology in identical older male twins. Neurology 52: 1119-1124. doi:10.1212/WNL.52.6.1119

Colby SL, Ortman JM. 2015. Projections of the size and composition of the US population: 2014 to 2060. Current Population Reports: 25-1143, U.S. Census Bureau document (https://files.eric.ed.gov/fulltext/ED578934 .pdf).

Corrada MM, Hayden KM, Paganini-Hill A, Bullain SS, DeMoss J, Aguirre C, Brookmeyer R, Kawas CH. 2017. Age of onset of hypertension and risk of dementia in the oldest-old: the 90+ study. Alzheimers Dement 13: 103110. doi:10.1016/j.jalz.2016.09.007

Cournot M, Marquié JC, Ansiau D, Martinaud C, Fonds H, Ferrières J, Ruidavets JB. 2006. Relation between body mass index and cognitive function in healthy middle-aged men and women. Neurology 67: 12081214. doi:10.1212/01.wnl.0000238082.13860.50

Crichton GE, Elias MF, Davey A, Alkerwi A. 2014. Cardiovascular health and cognitive function: The Maine-Syracuse Longitudinal Study. PLoS One 9: e89317. doi:10.1371/journal.pone.0089317

Daviglus ML, Talavera GA, Avilés-Santa ML, Allison M, Cai J, Criqui MH, Gellman M, Giachello AL, Gouskova N, Kaplan RC, et al. 2012. Prevalence of major cardiovascular risk factors and cardiovascular diseases among Hispanic/Latino individuals of diverse backgrounds in the United States. JAMA 308: 1775-1784. doi:10.1001/jama.2012 .14517

DeCarli C, Reed BR, Jagust W, Martinez O, Ortega M, Mungas D. 2008. Brain Behavior Relationships Among African Americans, Whites, and Hispanics. Alzheimer Dis Assoc Disord 22: 382-391. doi:10.1097/WAD .0b013e318185e7fe

Farrer LA, Cupples LA, Haines JL, Hyman B, Kukull WA, Mayeux R, Myers RH, Pericak-Vance MA, Risch N, van Duijn CM. 1997. Effects of age, sex, and ethnicity on the association between apolipoprotein $\mathrm{E}$ genotype and Alzheimer disease. JAMA 278: 1349. doi:10.1001/jama .1997.03550160069041

Fenelon A, Chinn JJ, Anderson RN. 2017. A comprehensive analysis of the mortality experience of Hispanic subgroups in the United States: Variation by age, country of origin, and nativity. SSM Popul Health 3: 245-254. doi:10.1016/j.ssmph.2017.01.011

Fisk JE, Sharp CA. 2004. Age-related impairment in executive functioning: updating, inhibition, shifting, and access. J Clin Exp Neuropsychol 26: 874-890. doi:10.1080/13803390490510680

Fitten LJ, Ortiz F, Ponton M. 2001. Frequency of Alzheimer's disease and other dementias in a community outreach sample of Hispanics. J Am Geriatr Soc 49: 1301-1308. doi:10.1046/j.1532-5415.2001.49257.x

Friedman NP, Miyake A. 2017. Unity and diversity of executive functions: Individual differences as a window on cognitive structure. Cortex 86: 186-204. doi:10.1016/j.cortex.2016.04.023

Fuentes E, Fuentes F, Vilahur G, Badimon L, Palomo I. 2013. Mechanisms of chronic state of inflammation as mediators that link obese adipose tissue and metabolic syndrome. Mediators Inflamm 2013: 136584. doi:10 $.1155 / 2013 / 136584$

Gallo LC, Penedo FJ, Espinosa de Los Monteros K, Arguelles W. 2009a. Resiliency in the face of disadvantage: Do Hispanic cultural characteristics protect health outcomes? J Pers 77: 1707-1746. doi:10 $.1111 / \mathrm{j} .1467-6494.2009 .00598 . \mathrm{x}$

Gallo LC, de Los Monteros KE, Allison M, Roux AD, Polak JF, Watson KE, Morales LS. 2009b. Do socioeconomic gradients in subclinical atherosclerosis vary according to acculturation level? Analyses of Mexican-Americans in the multi-ethnic study of atherosclerosis. Psychosom Med 71: 756-762. doi:10.1097/PSY.0b013e3181b0d2b4

Glymour MM, Manly JJ. 2008. Lifecourse social conditions and racial and ethnic patterns of cognitive aging. Neuropsychol Rev 18: 223-254. doi:10 1007/s11065-008-9064-z

Go AS, Mozaffarian D, Roger VL, Benjamin EJ, Berry JD, Borden WB, Bravata DM, Dai S, Ford ES, Fox CS, et al. 2013. Heart disease and stroke statistics-2013 update: A report from the American Heart Association. Circulation 127: e6-e245.

González HM, Tarraf W, Gouskova N, Gallo LC, Penedo FJ, Davis SM, Lipton RB, Argüelles W, Choca JP, Catellier DJ, et al. 2015. Neurocognitive function among middle-aged and older Hispanic/ Latinos: Results from the Hispanic community health study/study of Latinos. Arch Clin Neuropsychol 30: 68-77. doi:10.1093/arclin/acu066

González HM, Tarraf W, Vásquez P, Sanderlin AH, Rosenberg NI, Davis S, Rodríguez CJ, Gallo LC, Thyagarajan B, Daviglus M, et al. 2018. Metabolic syndrome and neurocognition among diverse middle-aged 
and older Hispanics/Latinos: HCHS/SOL results. Diabetes Care 41: 15011509. doi: $10.2337 / \mathrm{dc} 17-1896$

Graham NL, Emery T, Hodges JR. 2004. Distinctive cognitive profiles in Alzheimer's disease and subcortical vascular dementia. J Neurol Neurosurg Psychiatry 75: 61-71.

Gunstad J, Paul RH, Cohen RA, Tate DF, Spitznagel MB, Gordon E. 2007. Elevated body mass index is associated with executive dysfunction in otherwise healthy adults. Compr Psychiatry 48: 57-61. doi:10.1016/j .comppsych.2006.05.001

Gunstad J, Strain G, Devlin MJ, Wing R, Cohen RA, Paul RH, Crosby RD, Mitchell JE. 2011. Improved memory function 12 weeks after bariatric surgery. Surg Obes Relat Dis 7: 465-472. doi:10.1016/j .soard.2010.09.015

Gurland B, Wilder D, Lantigua R, Stern Y, Chen J. 1999. Rates of dementia in three enthnoracial groups. Int J Geriatr Psychiatry 493: 481-493.

Haan MN, Mungas DM, Gonzalez HM, Ortiz TA, Acharya A, Jagust WJ. 2003. Prevalence of dementia in older Latinos: The influence of type 2 diabetes mellitus, stroke and genetic factors. J Am Geriatr Soc 51: 169-177. doi:10 $.1046 / \mathrm{j} .1532-5415.2003 .51054 . \mathrm{x}$

Haan MN, Lee A, Odden MC, Aiello AE, To TM, Neuhaus JM. 2016. Gender Differences in the combined effects of cardiovascular disease and osteoarthritis on progression to functional impairment in older Mexican Americans. J Gerontol A Biol Sci Med Sci 71: 1089-1095. doi:10.1093/ gerona/glw014

Helzner EP, Scarmeas N, Cosentino S, Tang MX, Schupf N, Stern Y. 2008. Survival in Alzheimer disease: a multiethnic, population-based study of incident cases. Neurology 71: 1489-1495. doi:10.1212/01.wnl 0000334278.11022 .42

Jagust W, Harvey D, Mungas D, Haan M. 2005. Central obesity and the aging brain. Arch Neurol 62: 1545-1548. doi:10.1001/archneur.62.10.1545

Kannel WB, Wolf PA, McGee DL, Dawber TR, McNamara P, Castelli WP. 1981. Systolic blood pressure, arterial rigidity, and risk of stroke. The Framingham study. JAMA 245: 1225-1229. doi:10.1001/jama.1981 .03310370017013

Kiliaan AJ, Arnoldussen IAC, Gustafson DR. 2014. Adipokines: A link between obesity and dementia? Lancet Neurol 13: 913-923. doi:10.1016/ S1474-4422(14)70085-7

Lamar M, Wu D, Durazo-Arvizu RA, Brickman AM, Gonzalez HM, Tarraf W, Daviglus ML. 2017. Cognitive associates of current and more intensive control of hypertension: Findings from the Hispanic community health study/study of Latinos. Am J Hypertens 30: 624-631. doi:10.1093/ajh/ hpx023

LaRue A, Romero LJ, Ortiz IE, Lang HC, Lindeman RD. 1999. Neuropsychological performance of Hispanic and non-Hispanic older adults: an epidemiologic survey. Clin Neuropsychol 13: 474-486. doi:10 .1076/1385-4046(199911)13:04;1-Y;FT474

Levin BE, Llabre MM, Dong C, Elkind MSV, Stern Y, Rundek T, Sacco RL, Wright CB. 2014. Modeling metabolic syndrome and its association with cognition: the Northern Manhattan study. J Int Neuropsychol Soc 20: $951-960$. doi:10.1017/S1355617714000861

Li Y, Dai Q, Jackson JC, Zhang J. 2008. Overweight is associated with decreased cognitive functioning among school-age children and adolescents. Obesity 16: 1809-1815. doi:10.1038/oby.2008.296

Marcus J, Gardener H, Rundek T, Elkind MSV, Sacco RL, Decarli C, Wright CB. 2011. Baseline and longitudinal increases in diastolic blood pressure are associated with greater white matter hyperintensity volume: the Northern Manhattan study. Stroke 42: 2639-2641. doi:10.1161/ STROKEAHA.111.617571

Marquine MJ, Heaton A, Johnson N, Rivera-Mindt M, Cherner M, Bloss C, Hulgan T, Umlauf A, Moore DJ, Fazeli P, et al. 2018. Differences in neurocognitive impairment among HIV-infected Latinos in the United States. J Int Neuropsychol Soc 24: 163-175. doi:10.1017/ S1355617717000832

Medina-Inojosa J, Jean N, Cortes-Bergoderi M, Lopez-Jimenez F. 2014. The Hispanic paradox in cardiovascular disease and total mortality. Progr Cardiovasc Dis 57: 286-292. doi:10.1016/j.pcad.2014.09.001

Memel M, Bourassa K, Woolverton C, Sbarra DA. 2016. Body mass and physical activity uniquely predict change in cognition for aging adults. Ann Behav Med 50: 397-408. doi:10.1007/s12160-015-9768-2

Meng X-F, Yu J-T, Wang H-F, Tan M-S, Wang C, Tan C-C, Tan L. 2014. Midlife vascular risk factors and the risk of Alzheimer's disease: a systematic review and meta-analysis. J Alzheimer Dis 42: 1295-1310. doi:10.3233/JAD-140954

Miyake A, Friedman NP. 2012. The nature and organization of individual differences in executive functions. Curr Dir Psychol Sci 21: 8-14. doi:10 $.1177 / 0963721411429458$

Mungas D, Reed BR, Farias ST, Decarli C. 2009. Age and education effects on relationships of cognitive test scores with brain structure in demographically diverse older persons. Psychol Aging 24: 116-128. doi:10.1037/a0013421

Nilsson LG, Nilsson E. 2009. Overweight and cognition. Scand J Psychol 50: 660-667. doi:10.1111/j.1467-9450.2009.00777.x
Ogden CL, Carroll MD, Kit BK, Flegal KM. 2013. Prevalence of obesity among adults: United States, 2011-2012. NCHS Data Brief 131: $1-8$.

Pedditizi E, Peters R, Beckett N. 2016. The risk of overweight/obesity in mid-life and late life for the development of dementia: A systematic review and meta-analysis of longitudinal studies. Age Ageing 45: 14-21. doi:10.1093/ageing/afv151

Raz N, Rodrigue KM, Acker JD. 2003. Hypertension and the brain: vulnerability of the prefrontal regions and executive functions. Behav Neurosci 117: 1169-1180. doi:10.1037/0735-7044.117.6.1169

Reitan RM. 1958. Validity of the trail making test as an indicator of organic brain damage. Percept Mot Skills 8: 271-276. doi:10.2466/pms.1958.8.3 .271

Rodríguez-Saldaña J, Morley JE, Reynoso MT, Medina CA, Salazar P, Cruz E, Torres ALN. 2002. Diabetes mellitus in a subgroup of older Mexicans: prevalence, association with cardiovascular risk factors, functional and cognitive impairment, and mortality. J Am Geriatr Soc 50: 111-116. doi:10.1046/j.1532-5415.2002.50016.x

Ruiz JM, Steffen P, Smith TB. 2013. Hispanic mortality paradox: a systematic review and meta-analysis of the longitudinal literature. Public Health 103: e52-e60. doi:10.2105/AJPH.2012.301103

Ruiz JM, Hamann HA, Mehl MR, O'Connor M-F. 2016. The Hispanic health paradox: from epidemiological phenomenon to contribution opportunities for psychological science. Group Process Intergroup Relat 19: 462-476. doi:10.1177/1368430216638540

Ruiz JM, Sbarra D, Steffen PR. 2018. Hispanic ethnicity, stress psychophysiology and paradoxical health outcomes: A review with conceptual considerations and a call for research. Int J Psychophysiol 131: 24-29. doi:10.1016/j.ijpsycho.2018.04.001

Salat DH. 2014. Imaging small vessel-associated white matter changes in aging. Neuroscience 276: 174-186. doi:10.1016/j.neuroscience.2013.11 .041

Smith E, Hay P, Campbell L, Trollor JN. 2011. A review of the association between obesity and cognitive function across the lifespan: Implications for novel approaches to prevention and treatment. Obes Rev 12: 740755. doi:10.1111/j.1467-789X.2011.00920.x

Sorlie PD, Allison MA, Avilés-Santa ML, Cai J, Daviglus ML, Howard AG, Kaplan R, Lavange LM, Raij L, Schneiderman N, et al. 2014. Prevalence of hypertension, awareness, treatment, and control in the Hispanic community health study/study of Latinos. Am J Hypertens 27: 793-800. doi:10.1093/ajh/hpu003

Szoeke C, Lehert P, Henderson VW, Dennerstein L, Desmond P, Campbell S. 2016. Predictive factors for verbal memory performance over decades of aging: data from the women's healthy ageing project. Am J Geriatr Psychiatry 24: 857-867. doi:10.1016/j.jagp.2016.05.008

Tarraf W, Rodríguez CJ, Daviglus ML, Lamar M, Schneiderman N, Gallo L, Talavera GA, Kaplan RC, Fornage M, Conceicao A, et al. 2017. Blood pressure and Hispanic/Latino cognitive function: Hispanic community health study/study of Latinos results. J Alzheimer Dis 59: 31-42. doi:10 .3233/JAD-170017

Tarraf W, Jensen GA, Dillaway HE, Vasquez P, Gonzalez HM. 2018. Trajectories of Aging among US Older Adults: Mixed Evidence for a Hispanic Paradox. J Gerontol B Psychol Sci Soc Sci doi:10.1093/geronb/ gby057.

Tolppanen A-M, Solomon A, Soininen H, Kivipelto M. 2012. Midlife vascular risk factors and Alzheimer's disease: evidence from epidemiological studies. J Alzheimer Dis 32: 531-540. doi:10.3233/ JAD-2012-120802

Tomaszewski Farias S, Mungas D, Reed B, Haan MN, Jagust WJ. 2004. Everyday functioning in relation to cognitive functioning and neuroimaging in community-dwelling Hispanic and Non-Hispanic older adults. J Int Neuropsychol Soc 10: 342-354.

Tucker AM, Stern Y. 2011. Cognitive reserve in aging. Curr Alzheimer Res 8: 354-360. doi:10.2174/156720511795745320

van den Berg E, Kloppenborg RP, Kessels RPC, Kappelle LJ, Biessels GJ. 2009. Type 2 diabetes mellitus, hypertension, dyslipidemia and obesity: a systematic comparison of their impact on cognition. Biochim Biophys Acta 1792: 470-481. doi:10.1016/j.bbadis.2008.09.004

Verstynen TD, Weinstein A, Erickson KI, Sheu LK, Marsland AL, Gianaros PJ. 2013. Competing physiological pathways link individual differences in weight and abdominal adiposity to white matter microstructure. Neuroimage 79: 129-137. doi:10.1016/j.neuroimage .2013 .04 .075

Walther K, Birdsill AC, Glisky EL, Ryan L. 2010. Structural brain differences and cognitive functioning related to body mass index in older females. Hum Brain Mapp 31: 1052-1064. doi:10.1002/hbm.20916

Wechsler D. 1987. Wechsler Memory Scale - revised manual. The Psychological Corporation, San Antonio.

Weintraub S, Salmon D, Mercaldo N, Ferris S, Graff-Radford NR, Chui H, Cummings J, DeCarli C, Foster NL, Galasko D, et al. 2009. The Alzheimer's disease centers' data set (UDS): the neuropsychological test 
battery. Alzheimer Dis Assoc Disord 23: 91-101. doi:10.1097/WAD $.0 \mathrm{~b} 013 \mathrm{e} 318191 \mathrm{c} 7 \mathrm{dd}$

Weintraub S, Besser L, Dodge HH, Teylan M, Ferris S, Goldstein FC, Giordani B, Kramer J, Loewenstein D, Marson D, et al. 2018. Version 3 of the Alzheimer Disease Centers' neuropsychological test battery in the uniform data set (UDS). Alzheimer Dis Assoc Disord 32: 10-17. doi:10 .1097/WAD.0000000000000223

Wu CC, Mungas D, Petkov CI, Eberling JL, Zrelak PA, Buonocore MH, Brunberg JA, Haan MN, Jagust WJ. 2002. Brain structure and cognition in a community sample of elderly Latinos. Neurology 59: 383-391. doi:10.1212/WNL.59.3.383

Yaffe K, Haan M, Blackwell T, Cherkasova E, Whitmer RA, West N. 2007. Metabolic syndrome and cognitive decline in elderly Latinos: findings from the Sacramento Area Latino Study of Aging Study. J Am Geriatr Soc 55: 758-762. doi:10.1111/j.1532-5415.2007.01139.x

Yesavage JA, Kinoshita LM, Noda A, Lazzeroni LC, Fairchild JK, Taylor J, Kulick D, Friedman L, Cheng J, Zeitzer JM, et al. 2014. Effects of body mass index-related disorders on cognition: preliminary results. Diabetes Metab Syndr Obes 7: 145-151. doi:10.2147/DMSO.S60294

Yeung SE, Loken Thornton W. 2017. "Do it-yourself": home blood pressure as a predictor of traditional and everyday cognition in older adults. PLoS One 12: e0177424. doi:10.1371/journal.pone.0177424

Zahodne LB, Manly JJ, Narkhede A, Griffith EY, DeCarli C, Schupf NS, Mayeux R, Brickman AM. 2015. Structural MRI predictors of late-life cognition differ across African Americans, Hispanics, and Whites. Curr Alzheimer Res 12: 632-639. doi:10.2174/1567205012666150530203214

Zahodne LB, Manly JJ, Smith J, Seeman T, Lachman ME. 2017.

Socioeconomic, health, and psychosocial mediators of racial disparities in cognition in early, middle, and late adulthood. Psychol Aging 32: 118130. doi:10.1037/pag0000154

Received January 16, 2019; accepted in revised form May 10, 2019. 


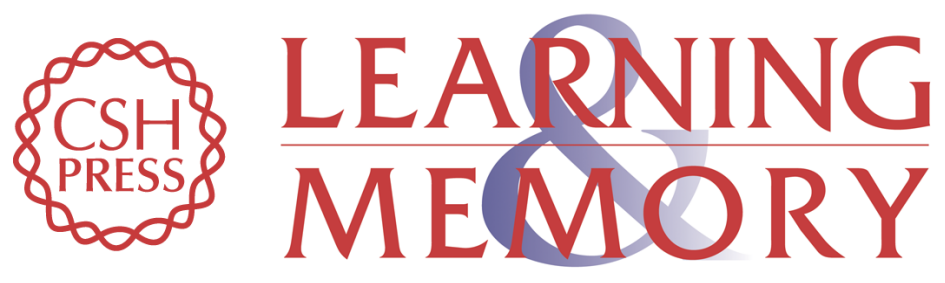

\section{The impact of cardiovascular risk factors on cognition in Hispanics and non-Hispanic whites}

Ariana Stickel, Andrew McKinnon, John Ruiz, et al.

Learn. Mem. 2019, 26:

Access the most recent version at doi:10.1101/Im.048470.118

References This article cites 77 articles, 8 of which can be accessed free at: http://learnmem.cshlp.org/content/26/7/235.full.html\#ref-list-1

Creative This article, published in Learning \& Memory, is available under a Creative Commons Commons License (Attribution-NonCommercial 4.0 International), as described at License http://creativecommons.org/licenses/by-nc/4.0/.

Email Alerting Receive free email alerts when new articles cite this article - sign up in the box at the Service top right corner of the article or click here. 